\title{
Entzündliche Muskelerkrankungen - Aktueller Stand zur Diagnostik und Therapie der Myositis
}

\section{Inflammatory Muscle Disorders - State of the Art of Diagnosis and Treatment of Myositis}

\author{
Autoren \\ Maren Fitzner ${ }^{1}$, Jens Schmidt ${ }^{1,2}$ \\ Institute \\ 1 Klinik für Neurologie, Universitätsmedizin Göttingen, Göttingen \\ 2 Institut für Neuroimmunologie, Institut für Multiple-Sklerose- \\ Forschung und Hertie Stiftung, Universitätsmedizin Göttingen
}

Schlüsselwörter

Myositis, inflammatorische Myopathien, Dermatomyositis, Polymyositis, Overlap-Syndrom, Nekrotisierende Myopathie, Einschlusskörpermyositis

Key words

myositis, inflammatory myopathies, dermatomyositis, polymyositis, overlap syndrome, necrotizing myopathy, inclusion body myositis

Korrespondenzadresse

Prof. Dr. Jens Schmidt

Klinik für Neurologie

Universitätsmedizin Göttingen

Göttingen

j.schmidt@gmx.org

Erstveröffentlichung

DOI http://dx.doi.org/10.1055/s-0042-102954

Akt Rheumatol 2016; 41: 232-242

(c) Georg Thieme Verlag KG Stuttgart · New York

ISSN 0341-051X

Bibliografie

DOI http://dx.doi.org/10.1055/s-0042-123877

Phys Med Rehab Kuror 2017; 27: 23-34

(c) Georg Thieme Verlag KG Stuttgart · New York

ISSN 0940-6689

\section{ZUSAMMENFASSUNG}

Bei den idiopathischen inflammatorischen Myopathien unterscheidet man die Polymyositis (PM), Dermatomyositis (DM), nekrotisierende Myopathie (NM), Einschlusskörpermyositis (inclusion body myositis = IBM) und Myositis bei Overlap-Syndrom. Es handelt es sich um eine heterogene Gruppe erworbener Muskelerkrankungen, die sich durch eine progrediente Muskelschwäche und Muskelatrophien auszeichnet. Die Abgrenzung der einzelnen Subtypen hat insbesondere im Hinblick auf die Prognose und Wahl der Therapie eine wesentliche Bedeutung. Die Identifikation muskelspezifischer Antikörper hat in den letzten Jahren die Diagnostik wesentlich vorangebracht, jedoch bleibt die Muskelbiopsie weiterhin ein unverzichtbares Hauptelement in der differentialdiagnostischen Abklärung von Myositissyndromen. Die Therapieempfehlungen basieren in Anbetracht des Mangels hochqualitativer Studien weiterhin überwiegend auf empirischen Daten und Expertenmeinungen und es sind erstmals fachübergreifende Leitlinien mit Beteiligung von Dermatologen, Rheumatologen und Neurologen entwickelt worden. Während bei der PM, DM und NM immunsuppressive Therapien zum Einsatz kommen, scheint die IBM gegenüber diesen Ansätzen weitestgehend refraktär zu sein. Hierdurch wird deutlich, dass auch die Abgrenzung zu anderen, nicht-entzündlichen Muskelerkrankungen essenziell ist. Die vorliegende Übersicht stellt den aktuellen state-of-the-art zur Diagnostik und Therapie von Myositiden dar.

\section{ABSTRACT}

Idiopathic inflammatory myopathies include polymyositis (PM), dermatomyositis (DM), necrotizing myopathy (NM), inclusion body myositis (IBM), and myositis in overlap syndromes. This is a heterogeneous group of acquired muscle diseases characterised by progressive muscle weakness and muscular atrophy. It is crucial to differentiate between the individual subtypes, especially with a view to prognosis and choice of treatment. Although the identification of muscle-specific antibodies has significantly advanced the diagnostic process in the past few years, muscle biopsy remains an indispensable element in the differentialdiagnostic workup of myositis syndromes. Due to the lack of highquality studies, most treatment recommendations continue to be based on empirical data and expert opinions, and the first interdisciplinary guidelines have been developed jointly by dermatologists, rheumatologists and neurologists. While patients with PM, DM and NM are eligible for immunosuppressive treatment, IBM seems to be largely refractory to this approach. This illustrates that it is also essential to distinguish between myositis and other, non-inflammatory muscle diseases. The following review article presents the current state-of-theart approach to the diagnostic workup and treatment of myositis.

\section{Einleitung}

Unter Myositiden sind eine Gruppe erworbener, inflammatorischer Myopathien zusammengefasst, die sich durch einen Verlust von Muskelkraft, Muskelatrophien, das mögliche Vorkommen von Myalgien und andere Begleitsymptome äußern. Man unterscheidet die 5 Subtypen Polymyositis (PM), Dermatomyositis (DM), nekrotisierende
Myopathie (NM), Myositis bei Overlap-Syndrom („Overlap Myositis“) und Einschlusskörpermyositis (inclusion body myositis, IBM). Anhand charakteristischer klinischer oder neuropathologischer Merkmale lassen sich die 5 Subtypen unterscheiden. Die klinischen Symptome wie Hautveränderungen bei der Dermatomyositis oder ein klares Verteilungsmuster der Muskelparesen und -atrophien liegt jedoch nicht 
bei jedem Patienten vor, so dass eine prompte Diagnose nicht immer einfach scheint. Die Muskelbiopsie und pathologische Untersuchung des Gewebes bleibt daher ein essentieller Bestandteil der differentialdiagnostischen Einordnung inflammatorischer Myopathien.

Die Ätiologie dieser Erkrankungen ist immer noch nicht abschließend geklärt und bleibt Gegenstand der Forschung. Im Zentrum der Pathogenese stehen autoimmunologische Prozesse. Eine Ausnahme stellt hier die IBM dar, bei der sich neuropathologisch neben inflammatorischen Vorgängen auch neurodegenerative Veränderungen nachweisen lassen.

Nicht selten zeigen einige Subtypen von inflammatorischen Myopathien eine Assoziation mit anderen Erkrankungen wie Kollagenosen, Malignomen oder Lungenerkrankung, die einen wesentlichen Einfluss auf die Morbidität haben können. Eine sorgfältige Einordnung der Diagnose und entsprechende Umfelddiagnostik ist daher obligat, insbesondere vor dem Hintergrund der Auswahl des Therapieregimes. Die Behandlung der inflammatorischen Myopathien konnte in den vergangenen Jahren optimiert werden. Aufgrund der Seltenheit der Erkrankungen basieren viele der in den Leitlinien empfohlenen Therapieregimes auf klinischer Erfahrung und Studien mit niedriger Evidenz. Während bei der PM, DM und NM immunsuppressive Substanzen erfolgreich zum Einsatz kommen, scheint die IBM gegenüber diesen Therapiestrategien resistent zu sein. Im Jahr 2015 sind erstmals über die Deutsche Gesellschaft für Neurologie fachübergreifende, deutschsprachige Therapieleitlinien erschienen, an denen Dermatologen, Rheumatologen und Neurologen mitgewirkt haben (www.awmf.org: Therapie der Myositis).

\section{Diagnostik von Myositiden}

Die Klassifikation der Myositiden erfolgt anhand der charakteristischen klinischen, histopathologischen und immunologischen Merkmale. Mittlerweile werden auch erweiterte laborchemische und bildgebende Verfahren in der Einteilung berücksichtigt und sind somit Bestandteil der standardisierten Diagnostik (Übersichten in: [1-3]). Frühere Diagnosekriterien wie von Bohan und Peter $[4,5]$, die auf rein klinischen Merkmalen, wie dem Vorhandensein oder Fehlen von typischen Hautveränderungen, zur Unterscheidung zwischen PM und DM beruhen, werden aus heutiger Sicht zunehmend als überholt eingestuft. Die Muskelbiopsie ist zentraler, unabdingbarer Bestandteil der Diagnostik von inflammatorischen Myopathien.

\section{Neurologische Untersuchung}

Alle Subtypen von inflammatorischen Myopathien führen zu einer progredienten Muskelschwäche, deren Verteilungsmuster jedoch variieren kann. Dabei finden sich in der klinisch-neurologischen Untersuchung zudem häufig auch umschriebene Muskelatrophien. Bei einigen Patienten bestehen Myalgien oder andere extramuskuläre Begleitsymptome, wie z. B. typische Hautveränderungen bei der Dermatomyositis oder Symptome, die auf eine weitere Organbeteiligung hinweisen. Es sollte daher eine ausführliche Anamneseerhebung sowohl im Hinblick auf die Dynamik und Verteilung der muskulären Beschwerden, als auch im Hinblick auf Begleiterscheinungen, wie Hautveränderungen, Luftnot, Malignom oder Kollagenosen erhoben werden. Eine sorgfältige Anamneseerhebung hinsichtlich des zeitlichen Verlaufs und Verteilungsmusters der Muskelbeschwerden kann die klinische Einordnung erleichtern. So weisen Patienten mit einer IBM einen langsameren Krankheitsverlauf mit eher asymmetrischer Verteilung und auch distaler Betonung der Paresen und Muskelatrophien auf, während bei der $\mathrm{PM}, \mathrm{DM}$ und NM eher ein proximal-symmetrisches Verteilungsmuster vorliegt und der Beginn eher subakut als chronisch ist. Es ist von grundlegender Bedeutung, dass eine Vielzahl an Muskelerkrankungen zu einer Muskelschwäche und CK-Erhöhung führen können und sowohl klinisch als auch histologisch ein Myositissyndrom „imitieren“ können. Eine sorgfältige klinische Untersuchung ist zur Differenzialdiagnose unabdingbar, da nur durch die Synthese aus klinischem Befund, laborchemischem Befund und Muskelbiopsiebefund eine verlässliche Diagnose ermöglicht.

Da inflammatorische Myopathien nicht selten mit einer Dysphagie einhergehen, sollte auch hiernach explizit gefragt werden, ggf. unter Zuhilfenahme standardisierter Fragebögen. Darüber hinaus erfolgt neben einer eingehenden allgemeinen körperlichen Untersuchung insbesondere eine detaillierte Beschreibung der Muskeltrophik und Einteilung der Muskelparesen entsprechend der Kraftgrade (z. B. nach dem Manual Muscle Testing 8 [MMT-8]). Vor allem auch im Hinblick auf die weiteren Verlaufskontrollen ist eine sorgfältige Dokumentation essentiell, um Therapieeffekte möglichst objektiv beurteilen zu können. Die anamnestische Erhebung der Gehstrecke bzw. auch dessen formale Testung ist hierbei als zusätzlicher Parameter vor allem auch im mittelfristigen und langfristigen Verlauf sehr hilfreich.

\section{Laborchemische Untersuchungen}

Die laborchemischen Untersuchungen umfassen zunächst Routineparameter wie Blutbild, Blutsenkungsgeschwindigkeit, CRP, LDH, AST, ALT und insbesondere die CK. Nicht selten ist bei akut verlaufenden inflammatorischen Myopathien der CK-Wert bis auf das 50 fache angestiegen und lässt auf das Ausmaß des Muskelzerfalls rückschließen. Jedoch ist es wichtig, dass eine regelrechte CK eine Myositis oder andere Myopathie nicht ausschließt. Eine Normalisierung der CK kann z. B. auch im Verlauf der Erkrankung durch Regredienz der Entzündung mitbedingt werden.

Aufgrund der häufigen Assoziation mit anderen Kollagenosen sollte darüber hinaus eine entsprechende Bestimmung des Rheumafaktors, ANA, ENA und ANCA erfolgen.

Einen noch größeren Stellenwert nimmt mittlerweile die Bestimmung der sogenannten „Myositis-spezifischen“ bzw. „Myositisassoziierten“ Auto-Antikörper ein, auf die im Folgenden separat eingegangen wird.

\section{„Myositis-spezifische“ bzw. „Myositis-assoziierte“ Auto-Antikörper}

In den letzten Jahren konnten verschiedene Antikörper identifiziert werden, die in der Diagnostik von inflammatorischen Myopathien zunehmende Bedeutung erlangt haben. Die Einordnung als „Myositis-spezifische“ bzw. „Myositis-assoziierte“ Auto-Antikörper ist arbiträr und wird wegen zunehmend beschriebener Überschneidungen langfristig vermutlich nicht mehr sinnvoll sein.

Muskel-assoziierte Antikörper finden sich im Rahmen von systemischen Kollagenosen mit begleitender inflammatorischer Myopathie: Myositis bei Overlap-Syndrom („Overlap Myositis“). Bei einigen Patienten lassen sich „Myositis-spezifische“ Auto-Antikörper 
finden, die mit einer relativ hohen Spezifität mit dem Vorliegen einer der Myositis-Syndrome assoziiert sind. Ziel-Antigene dieser Antikörper sind häufig zytoplasmatische oder nukleäre Enzyme und Membranproteine, die an der Transkription und Proteinsynthese beteiligt sind. Sie können zur differentialdiagnostischen Einordnung hinzugezogen werden, reichen jedoch keineswegs aus, um alleinig eine Diagnose zu belegen. Von der Höhe der Antikörper-Titer lässt sich nicht direkt auf die Krankheitsaktivität rückschließen, so dass die Bestimmung zur Verlaufskontrolle oder Therapiemonitoring nicht unbedingt sinnvoll erscheint.

Mit am häufigsten finden sich Antisynthetase-Antikörper, die gegen verschiedene Aminoacyl-tRNA-Synthetasen gerichtet sind. Hierzu gehört der Jo-1-Antikörper, der sich bei bis zu 10-30\% der Patienten mit Anti-Synthetase-Syndrom findet, bei dem die Myositis begleitet wird von einer interstitiellen Pneumonitis, RaynaudSymptomatik und Arthritis.

Andere muskel-spezifische Antikörper können ebenfalls auf einen spezifischen Myositis-Subtyp hinweisen. So findet sich z. B. für Mi-2 Antikörper, die gegen eine nukleäre Helikase gerichtet sind, in 20-30\% eine Assoziation mit dem Vorliegen einer Dermatomyositis. Anti-P155/140 Antikörper lassen sich ebenfalls häufig bei Patienten mit DM und juveniler DM finden. Sie sind beim Erwachsenen in 50-75\% mit dem Vorliegen eines Malignoms und bei der juvenilen DM häufig mit schweren Hauterscheinungen assoziiert [6]. Bei der amyopathischen DM finden sich hingegen bei bis zu 50 \% der Patienten Anti-CADM140 Antikörper gegen das MDA-5 (melanoma differentiation associated gene 5). Diese weisen auf einen ungünstigeren Verlauf der Erkrankung bei hoher Assoziation mit einer schweren interstitiellen Lungenerkrankung hin [7].

Bei Patienten mit NM lassen sich häufig Antikörper gegen das signal recognition peptide (SRP) nachweisen. Zudem konnte ein weiterer Antikörper identifiziert werden, der sich gegen die HMGCoA-Reduktase richtet. Letzterer ist häufiger bei Patienten zu finden, die nach einer Statineinnahme eine NM entwickelt haben, kann aber auch ohne vorherige Statineinnahme vorliegen [8]. Für die IBM konnte mittlerweile auch ein Auto-Antikörper identifiziert werden. Er richtete sich gegen die zytosolische 5'Nukleotidase $1 \mathrm{~A}$ (Anti-Mup44) und lässt sich bei bis zu $60 \%$ der IBM-Patienten nachweisen [9, 10]. Die „Myositis assoziierten“ Auto-Antikörper werden im Abschnitt Myositis bei Overlap-Syndrom besprochen und eine Übersicht der wichtigsten „Myositis-spezifischen“ und „Myositisassoziierten“ Auto-Antikörper ist in \ Tab. 1 zusammengefasst.

\section{Neurophysiologische Testung (EMG und Neurografien)}

In der Elektromyografie (EMG) finden sich oft Fibrillationen und positive scharfe Wellen als Zeichen der pathologischen Spontanaktivität. Darüber hinaus zeigen sich bei Willkürinnervation myopathische Veränderungen in Form von verkürzten, polyphasischen und niedrigamplitudigen Potenzialen der motorischen Einheiten. Die EMG und Bestimmung der CK haben in der klinischen Diagnostik insbesondere einen Stellenwert in der Abgrenzung von Myopathien gegenüber anderen neuromuskulären Erkrankungen. Im Hinblick auf die weitere Diagnostik mittels Muskelbiopsie und um fehlerhafte Ergebnisse zu vermeiden, sollte die EMG-Untersuchung nicht im Muskel erfolgen, der für die Biopsie geplant ist. Ein fehlender Nachweis myopathischer Veränderungen oder florider Schädi- gung schließt eine Myositis nicht aus. Mindestens bei Patienten mit distaler Schwäche sollten auch Neurografien durchgeführt werden, da auch eine akut-progrediente Neuropathie eine CK-Erhöhung bewirken kann.

\section{Muskel-MRT}

Das MRT hat in der Diagnostik von Muskelerkrankungen in den letzten Jahren zunehmend an Stellenwert gewonnen. Im Muskel-MRT kann das die Entzündung begleitende Ödem insbesondere mithilfe der T2- und fettunterdrückten STIR-Sequenzen (short tau inversion recovery) dargestellt werden. In den genannten Sequenzen findet sich ein hyperintenses Signal in den ödematös veränderten Arealen. Untersuchungen haben gezeigt, dass die Signalintensität gut mit der Muskelinflammation, - nekrose und -degeneration und dem Ausmaß an Krankheitsaktivität korrelieren [11, 12]. Andere Sequenzen können hingegen den meist therapeutisch nicht mehr beeinflussbaren fettigen oder bindegewebigen Umbau des Muskelgewebes darstellen (T1-Wichtung).

So lässt sich bildgebend zum einen das Verteilungsmuster der beteiligten Muskeln darstellen, zum anderen kann das MRT bei der Auswahl eines geeigneten Biopsiemuskels hilfreich sein. Die Gabe von Kontrastmittel kann zudem einen Hinweis hinsichtlich der Krankheitsaktivität geben und somit auch als ein zusätzlicher Parameter zur Therapie- und Verlaufskontrolle genutzt werden.

\section{Muskelbiopsie}

In der Diagnostik von Myositiden stellt die Muskelbiopsie die wichtigste Untersuchung zum Nachweis einer Entzündung im Muskel dar sowie zur Einordnung des Subtyps und differenzialdiagnostischen Abgrenzung gegenüber anderen Myopathien oder neuromuskulären Erkrankungen. Die Biopsie sollte nach Möglichkeit immer vor Beginn einer immunsuppressiven Therapie erfolgen. Eine Untersuchung unter laufender Therapie liefert falsche Ergebnisse und ist nicht sicher zu verwerten. Nach bereits begonnener Immunsuppression ist daher immer eine Therapiepause vor geplanter Muskelbiopsie notwendig und bedeutet eine unnötige Verzögerung für den Patienten.

Darüber hinaus ist auch die sorgfältige Auswahl der Biopsiestelle wichtig, vor allem auch um falsch-negative Ergebnisse möglichst zu vermeiden. Der für die Biopsie ausgewählte Muskel sollte eine mittelgradige Parese aufweisen und mindestens 2 Wochen vor der Biopsieentnahme nicht myografisch untersucht worden sein. Vor geplanter Muskelbiopsie kann ein Muskel-MRT der entsprechenden Körperregion hilfreich sein, um einen geeigneten Muskel auszuwählen. Ein Muskel, der bereits stark fettig oder bindegewebig umgebaut ist, ist für eine Biopsie nicht mehr geeignet.

Die Biopsie sollte unter Lokalanästhesie über einen offenen Hautschnitt erfolgen. Anschließend erfolgt eine sorgfältige pathologische Untersuchung im Hinblick auf das Vorliegen entzündlicher, vaskulärer, myopathischer und bindegewebiger Veränderungen. Nach Möglichkeit sollte die histopathologische Untersuchung an einem etablierten Muskelzentrum durchgeführt werden. Anstelle eines Probenversandes, der zusätzliche Artefakte bedingen kann, empfiehlt sich die Vorstellung des Patienten in einem entsprechenden Zentrum, um die Biopsie durchführen zu lassen. Durch eine möglichst standardisierte Untersuchung des Muskelgewebes, vor allem auch unter gezielter Berücksichtigung des in- 
- Tab. 1 Übersicht der wichtigsten „Myositis-assoziierten“ und „Myositis-spezifischen Antikörper“.

\begin{tabular}{|c|c|c|c|}
\hline $\begin{array}{l}\text { „Myositis-spezifische } \\
\text { Antikörper“ }\end{array}$ & Zielantigen & Häufigkeit (\%) & Assoziierte Syndrome/Merkmale \\
\hline Anti-Synthetase-Antikörper & Aminoacyl-tRNA-Synthetase & & \multirow{9}{*}{ Anti-Synthetase-Syndrom } \\
\hline Anti-jo1 & Histidyl & $20-25$ & \\
\hline Anti-Zo & Phenylalanyl & \multirow{7}{*}{$1-5$} & \\
\hline Anti-Ha & Tyrosyl & & \\
\hline Anti-KS & Asparaginyl & & \\
\hline Anti-OJ & Isoleucyt & & \\
\hline Anti-E] & Glycyl & & \\
\hline Anti-PL12 & Alanyl & & \\
\hline Anti-PL7 & Threonyl & & \\
\hline Anti-Mi2 & nukleare Helikase & $5-10$ & klassisches Bild der DM \\
\hline Anti-P155/140 & TIF1-Y & $\begin{array}{c}\sim 20 \text { bei DM } \\
\sim 23 \% \text { bei JDM }\end{array}$ & $\begin{array}{l}\text { DM: } 50-75 \% \text { Malignome, seltener interstitielle } \\
\text { Lungenerkrankung juvenile DM: schwere } \\
\text { Hauterscheinungen }\end{array}$ \\
\hline Anti-SRP & SRP & $\sim 5$ & NM \\
\hline Anti-CADM140 & $\begin{array}{l}\text { melanoma differentiation associated } \\
\text { gene } 5 \text { (MDA-5) }\end{array}$ & 50 (bei ADM) & $\begin{array}{l}\text { Amyopathische DM, rasch progrediente interstitiel- } \\
\text { le Lungenbeteiligung mit ungünstiger Prognose }\end{array}$ \\
\hline Anti-p140 & Kernprotein NXP2 & $<5$ & $\begin{array}{l}\text { juvenile DM mit Kalzinose, bei Erwachsenen } \\
\text { interstitielle Lungenerkrankung }\end{array}$ \\
\hline Anti-HMGCR & HMGCo-A-Reduktase & bis $60 \%$ & NM, insbesondere nach Statin-Exposition \\
\hline Anti-Mup44/cN1A & zytosolische 5'-Nukleotidase $1 \mathrm{~A}$ & $60 \%$ & IBM \\
\hline \multicolumn{4}{|c|}{ „Myositis-assoziierte Antikörper“ } \\
\hline Anti-U1RNP & $\begin{array}{l}\text { U1 small nuclear ribonucleoprotein } \\
\text { particles (Spliceosom) }\end{array}$ & $10 \%$ & Kollagenosen, Sklerodermie und SLE \\
\hline Anti-U2RNP & U2 small nuclear ribonucleoprotein & $15 \%$ & $\begin{array}{l}\text { Overlap von Myositis und Sklerodermie, Sjögren- } \\
\text { Syndrom }\end{array}$ \\
\hline Anti-SSA/Ro & $\begin{array}{l}\text { humane zytoplasmatische Ribonuk- } \\
\text { leoprotein-Komplex }\end{array}$ & bis $30 \%$ & $\begin{array}{l}\text { Bei Jo-1 positiven Patienten höheres Risiko für } \\
\text { schwere interstitielle Lungenerkrankung }\end{array}$ \\
\hline Anti-SSB/La & $\begin{array}{l}\text { Transkriptionsfaktor der RNA-Poly- } \\
\text { merase III }\end{array}$ & bis $14 \%$ & Sjögren-Syndrom \\
\hline Anti-Ku & DNA-abhängige Proteinkinase & $20-30 \%$ & Overlap von Myositis und Sklerodermie \\
\hline Anti-PmScl & Exoribonuklease & $\sim 10 \%$ & Overlap von Myositis und Sklerodermie \\
\hline
\end{tabular}

dividuellen klinischen Befundes, kann das nicht geringe Risiko einer Fehldiagnose erheblich reduziert werden.

\section{Erweiterte Diagnostik}

Eine Vielzahl der Patienten mit Myositissyndromen zeigen eine extramuskuläre Organbeteiligung oder Assoziation mit Tumorerkrankungen. Sowohl bei der Anamneseerhebung als auch der eingehenden körperlichen Untersuchung sollte dies bereits berücksichtigt werden. Lediglich bei der IBM ist bisher eine Tumorassoziation nicht beobachtet worden.

Bei der extramuskulären Organbeteiligung stellen insbesondere die interstitielle Pneumonie oder Lungenfibrose einen schwerwiegenden Bestandteil der Erkrankung dar. Auch Overlap-Syndrome mit anderen Kollagenosen sind häufig und äußern sich bspw. im Auftreten von Fieber, Raynaud-Symptomatik, Arthralgien oder unspezifischen Hautveränderungen.
Bis zu $30 \%$ der Dermatomyositis-Patienten und 15\% der Polymyositis-Patienten weisen eine assoziierte Tumorerkrankung auf. Dabei kann sich die Malignomerkrankung sowohl vor als auch nach dem Auftreten der Myositis entwickeln.

In Anbetracht der oben genannten Begleiterkrankungen sollte daher bei jedem Patienten mit einem Myositissyndrom unter Berücksichtigung der klinischen Symptomatik eine ausführliche Umfelddiagnostik durchgeführt werden. Hierzu zählen Röntgen- oder CTUntersuchungen der Lunge, eine Lungenfunktionsdiagnostik, EKG, Echokardiografie, erweiterte Labordiagnostik im Hinblick auf das Vorliegen anderer Kollagenosen sowie eine ausführliche Tumorsuche.

Da eine Vielzahl von Patienten mit Myositissyndromen zudem eine Dysphagie aufweisen, sollte neben einer entsprechend spezifischen Anamnese auch eine Untersuchung mittels flexibler Endoskopie (FEES) oder Videofluoroskopie stattfinden. 
- Tab. 2 Klinische und diagnostische Charakteristika der PM, DM, NM und IBM modifiziert nach der DGN-Leitlinie „Myositissyndrome“ www.dgn.org.

\begin{tabular}{|c|c|c|c|c|}
\hline Merkmale & PM & DM & NM & IBM \\
\hline Frauen: Männer & $2: 1$ & $2: 1$ & $1: 1$ & $1: 3$ \\
\hline Alter bei Erkrankung & $>18$ Jahre & 5-15 und 45-65 Jahre & $>18$ Jahre & >50 Jahre \\
\hline Krankheitsverlauf & akut - subakut & akut - subakut & akut - subakut & chronisch > 6 Monate \\
\hline Paresen & $\begin{array}{l}\text { proximal > distal } \\
\text { symmetrisch }\end{array}$ & $\begin{array}{l}\text { proximal > distal } \\
\text { symmetrisch }\end{array}$ & $\begin{array}{l}\text { proximal > distal } \\
\text { symmetrisch }\end{array}$ & $\begin{array}{l}\text { proximal = distal asymmetrisch, } \\
\text { Prädilektion: Fingerflexoren und M. } \\
\text { quadriceps }\end{array}$ \\
\hline Hautveränderungen & nein & ja & nein & nein \\
\hline Muskelatrophien & + & $(+)$ & + & ++ \\
\hline CK & bis 50 -fach & normal bis 50-fach & bis 50 -fach & normal bis $<15 \times$ normal \\
\hline Muskelbiopsie & $\begin{array}{l}\text { endomysiales Infiltrat } \\
\text { mit zytotoxischen } \\
\text { T-Zellen, die } \\
\text { Muskelfasern } \\
\text { umstellen, Nekrosen }\end{array}$ & $\begin{array}{l}\text { perifaszikuläre Atrophie, } \\
\text { perimysiale und perivasku- } \\
\text { läre entzündliche Infiltrate; } \\
\text { Komplementablagerung } \\
\text { um Kapillaren }\end{array}$ & $\begin{array}{l}\text { ausgedehnte Fasernek- } \\
\text { rosen, sekundäre } \\
\text { Makrophageninfiltrati- } \\
\text { on, keine wesentliche } \\
\text { T-Zell-Infiltration }\end{array}$ & $\begin{array}{l}\text { Entzündung wie bei PM und zusätzlich } \\
\text { Myodegeneration mit Faseratrophien, } \\
\text { geränderten Vakuolen und eosinophi- } \\
\text { len Einschlüssen }\end{array}$ \\
\hline Immunhistologie & $\begin{array}{l}\text { CD8 > CD4 T-Zellen, } \\
\text { Makrophagen, MHC-I }\end{array}$ & $\begin{array}{l}\text { CD20-immunreaktive } \\
\text { B-Zellen, Makrophagen, } \\
\text { CD4-Zellen MHC-I } \\
\text { perifaszikulär, Komplement }\end{array}$ & Makrophagen & $\begin{array}{l}\text { CD8 > CD4 T-Zellen, Makrophagen, } \\
\text { MHC-I } \\
\beta \text {-Amyloid und andere Neurodegene- } \\
\text { rations-assoziierte Proteine }\end{array}$ \\
\hline $\begin{array}{l}\text { Ansprechen auf } \\
\text { Immuntherapie }\end{array}$ & ja & ja & meistens ja & nicht oder minimal bzw. nur zeitweise \\
\hline assoziierte Probleme & $\begin{array}{l}\text { interstitielle } \\
\text { Lungenerkrankung, } \\
\text { Malignome } \\
\text { Myokarditis, andere } \\
\text { Organbeteiligung } \\
\text { bzw. systemische } \\
\text { Entzündung. }\end{array}$ & $\begin{array}{l}\text { interstitielle Lungenerkran- } \\
\text { kung, Malignome, } \\
\text { Vaskulitis, Myokarditis, } \\
\text { andere Organbeteiligung } \\
\text { bzw. systemische } \\
\text { Entzündung. }\end{array}$ & $\begin{array}{l}\text { kann Statin-induziert } \\
\text { sein; Malignome, } \\
\text { andere Organbeteili- } \\
\text { gung bzw. systemische } \\
\text { Entzündung. }\end{array}$ & Neuropathie (mild, sensibel) \\
\hline
\end{tabular}

\section{Differenzialdiagnosen von Myositiden}

Zur Differenzialdiagnose von Myositiden gehören Muskeldystrophien ebenso wie infektiöse, toxische oder metabolische Myopathien. Wichtige Unterscheidungsmerkmale sind: 1) der Zeitverlauf, 2) das Verteilungsmuster der Paresen und 3) die Labordiagnostik und der histologische Befund (Übersicht in > Tab. 2). Während sich PM/DM/NM vor allem subakut bzw. akut entwickeln, handelt es sich bei der IBM ebenso wie bei anderen Myopathien meistens um chronische Verläufe. Es ist zu betonen, dass das proximale Verteilungsmuster von Paresen nicht nur bei PM/DM/NM vorkommt, sondern auch bei metabolischen Myopathien und verschiedenen Muskeldystrophien, deren Erkrankungsbeginn auch manchmal erst in der 4. oder 5 Lebensdekade oder später liegt. Hinweise zur differenzialdiagnostischen Einordnung können sich somit aus der Anamnese, der Höhe der CK-Werte und entsprechender Enzym- und Erregerdiagnostik ergeben. Einen genauen Aufschluss liefert jedoch in der Regel die Muskelbiopsie. Die Muskelbiopsie lässt häufig eine Differenzierung der einzelnen Myositissyndrome zu, jedoch gibt es eine Reihe von Fehlerquellen: 1) eine (unspezifische) Entzündung kommt in der Muskelbiopsie bei einer Reihe von Muskeldystrophien vor; 2) In Abhängigkeit der lokalen Entzündungsschwere in einem bestimmten Muskel kann das Ausmaß der entzündlichen Infiltrate falsch niedrig sein; 3 ) Auch bei Myositiden gibt es mitunter degenerative Veränderungen im Muskel wie z. B. die Entstehung von Vakuolen, die bei zufälligem Überwiegen in einer Biopsie möglicherweise überbewertet werden und die adäquate Diagnose einer Myositis verhindern bzw. verzögern.

\section{Klassifikation der Myositiden}

Die Unterscheidung der Myositissyndrome erfolgt anhand klinischer, pathologischer und immunhistochemischer Kriterien in die Subtypen Dermatomyositis, Polymyositis, nekrotisierende Myopathie, Einschlusskörpermyositis und Myositis bei Overlap-Syndrom. Letzteres besteht aus einem Zusammenhang von entzündlichen Muskelveränderungen wie bei der DM oder PM und gleichzeitigem Bestehen anderer Organmanifestation bzw. Überlappung mit anderen Kollagenosen. In den letzten Jahren wird die Overlap-Myositis zunehmend als eigene Entität verstanden, so dass auch hier eine gesonderte Abhandlung erfolgen soll. Eine Übersicht über die Charakteristika der einzelnen Subtypen findet sich in $₹$ Tab. $\mathbf{2}$.

\section{Dermatomyositis}

Die Dermatomyositis tritt sowohl im Erwachsenen- als auch im Kindesalter (juvenile DM) auf und stellt die häufigste inflammatorische Myopathie dar. Erwachsene erkranken häufig zwischen dem 45. 
und 66. Lebensjahr bei einem Geschlechterverhältnis von Frauen zu Männern von 2:1. Sie zeichnet sich neben einer progredienten Muskelschwäche durch charakteristische Hautveränderungen auf, die der Erkrankung ihren Namen geben. Patienten weisen dabei ein photosensitives, fliederfarbenes Erythem mit ödematösen Schwellungen im Gesicht auf. Auch andere sonnenexponierte Stellen wie das Dekolletee, der Nacken, sowie Knie und Ellbogen und insbesondere die Hände sind häufig betroffen. Im Bereich des Handrückens finden sich zudem rötlich-livide Papeln und Plaques (sog. GottronPapeln) sowie eine rissige, verdickte und trockene Haut („Mechanikerhände“). Das Nagelbett weist Teleangiektasien und eine verdickte Nagelhaut auf und insbesondere bei der juvenilen Dermatomyositis finden sich subkutane Kalizifikationen, die Ulzerationen und Infektionen verursachen können [13].

Die Hautveränderungen können den muskulären Symptomen voraus gehen oder sich erst im Verlauf entwickeln. In der Diagnostik von inflammatorischen Myopathien sollte im Hinblick auf die DM immer eine konsiliarische Untersuchung durch einen Dermatologen erfolgen, der sich mit autoimmun-entzündlichen Erkrankungen auskennt.

Die muskulären Symptome entwickeln sich meist perakut innerhalb von Wochen bis Monaten und zeigen ein proximal-symmetrisches Verteilungsmuster. Patienten klagen typischerweise über Schwierigkeiten beim Treppensteigen und Aufstehen aus der Hocke. Im Verlauf treten auch Muskelatrophien auf. Nicht selten kommt es zu einer Beteiligung der Pharynxmuskulatur, die zu einer Dysphagie führt.

In bis zu 20\% der Fälle zeigen Patienten die typischen Hautveränderungen der Dermatomyositis, ohne dass sich jedoch eine muskuläre Beteiligung nachweisen lässt $[14,15]$. In diesen Fällen spricht man von einer amyopathischen Dermatomyositis oder „Dermatomyositis sine myositis“. Da eine subklinische Muskelbeteiligung vorliegen kann bzw. sich die muskulären Symptome auch erst im Verlauf einstellen können, sollte eine regelmäßige klinische Reevaluation erfolgen. Insbesondere die Abgrenzung zum systemischen Lupus erythematodes ist wichtig.

Die amyopathische DM ist auch mit dem Auftreten einer interstitiellen Pneumonie oder Lungenfibrose assoziiert, welche die Morbidität der Patienten erheblich erhöhen kann. Darüber hinaus findet man bei der DM häufig auch eine kardiale Beteiligung mit Perikardiatis und Kardiomyopathie. Die Überlappung mit anderen Kollagenosen ist wie bei der Polymyositis häufig und wird mittlerweile als Myositis bei Overlap-Syndrom („Overlap Myositis“) als weitere Subgruppe der inflammatorischen Myopathien betrachtet.

Im Hinblick auf die häufige Assoziation von Dermatomyositis mit Malignomen sollte eine ausführliche Auschlussdiagnostik erfolgen. Häufige Tumore, die mit einer Dermatomyositis assoziiert sind, sind das Mammakarzinom, Ovarialkarzinom, Melanome und Non-Hodgkin-Lymphome.

In der Routinediagnostik weisen DM-Patienten einen bis zu 50fach über der Norm erhöhten CK-Wert auf. Das EMG kann myopathische Veränderungen und pathologische Spontanaktivität aufweisen. Die MRT-Untersuchung kann aktive entzündliche Veränderungen dokumentieren.

In der Muskelbiopsie finden sich perivaskuläre und perimysiale entzündliche Infiltrate neben Muskelfasernekrosen, einer perifaszikulären Atrophie und regenerierenden Muskelfasern. Die entzündlichen Infiltrate bestehen überwiegend aus CD4-positiven
T-Zellen, B-Zellen sowie Makrophagen. Pathophysiologisch wird eine ischämische Muskelfaserschädigung durch entzündliche Veränderungen der Endothelzellen und Kapillaren angenommen [16]. Als alternativer Erklärungsansatz wird dem Interferon Typ 1 eine pathophysiologische Rolle bei der DM zugewiesen [17]. Insgesamt ist die Ätiologie der DM jedoch nicht geklärt.

\section{Polymyositis}

Frühere Klassifikationskriterien unterschieden allein anhand des Vorhandenseins von Hautveränderungen zwischen DM und PM. Nach heutigem Kenntnisstand wurde die PM daher häufig fehl- und überdiagnostiziert. Vielmehr handelte es sich meist um Patienten mit IBM oder NM. Unter den inflammatorischen Myopathien stellt die PM als alleinstehende Diagnose den seltensten Subtyp und eine Ausschlussdiagnose dar [1,2].

Die Erkrankung manifestiert sich im Erwachsenenalter und betrifft, wie die DM, häufiger Frauen als Männer im Verhältnis 2:1. Patienten berichten über das subakute Auftreten proximal betonter, symmetrischer Paresen, die im Verlauf zu Muskelatrophien der betroffenen Muskeln führen können. Oft werden auch begleitende Myalgien beschrieben, die jedoch nicht im Vordergrund der Beschwerdesymptomatik stehen.

Laborchemisch lässt sich üblicherweise eine deutliche Erhöhung der CK auf bis zum 50-fachen der Norm nachweisen. Wie bei der DM kann das EMG akute und chronische myopathische Veränderungen aufweisen. Die Muskelbiopsie ist wie auch bei der Dermatomyositis essentieller Bestandteil der Diagnostik. Auf eine hilfreiche MRT-Bildgebung zur Auswahl der entsprechenden Biopsiestelle ist oben bereits hingewiesen worden.

Histopathologisch weisen gesunde Muskelfasern charakteristischerweise eine Infiltration durch CD8 + T-Zellen auf. Daneben finden sich deutliche Muskelfasernekrosen. Immunhistologisch lassen sich eine deutliche, perifaszikuläre Expression von MHC-I auf nicht-nekrotischen Fasern sowie Komplementablagerungen nachweisen. Insbesondere auch zur Abgrenzung der PM gegenüber Muskeldystrophien ist die Muskelbiopsie unverzichtbar.

Pathophysiologisch handelt es sich bei der PM im Gegensatz zur DM um eine zellulär vermittelte Immunreaktion. Die CD8 + T-Zellen können MHC-I-Antigen präsentierende gesunde Muskelfasern umzingeln oder sogar infiltrieren. Durch proinflammatorische Chemokine und Zytokine wie IFN- $\gamma$ und TNF- $\alpha$ werden die myotoxischen Effekte noch verstärkt [18].

\section{Nekrotisierende Myopathie}

Die nekrotisierende Myopathie stellt eine eigene Entität dar und kommt deutlich häufiger vor als PM. Klinisch ist die NM jedoch nicht von PM zu unterscheiden und zeichnet sich ebenfalls durch proximal betonte, symmetrische Muskelparesen aus. Die Erkrankung kann in jedem Alter auftreten, manifestiert sich jedoch meist im mittleren bis höheren Erwachsenenalter.

Patienten berichten typischerweise über eine perakut (Tage) bis subakut (Wochen) auftretende Muskelschwäche, die in bis zu $80 \%$ der Fälle von Myalgien begleitet wird. Laborchemisch lassen sich meist deutlich erhöhte CK-Werte dokumentieren. Das EMG kann myopathische Veränderungen und floride Schädigungszeichen aufweisen und im MRT mitunter nekrotisches Muskelgewebe darstellen. 
Im Gegensatz zur PM lassen sich in der Muskelbiopsie keine primär entzündlichen Veränderungen nachweisen. Vielmehr finden sich ausgedehnte Muskelfasernekrosen mit sekundärer Infiltration durch Makrophagen [19]. Dennoch werden auch bei dieser inflammatorischen Myopathie entzündliche Mechanismen als wesentlicher Faktor in der Pathogenese angenommen. Die Hypothese wird unterstützt von der Tatsache, dass sich bei vielen Patienten Komplementablagerungen entlang der Gefäße nachweisen lassen.

Die NM kann im Anschluss an Viruserkrankungen, assoziiert mit Tumorerkrankungen, Kollagenosen oder assoziiert mit vorheriger Einnahme eines Statins auftreten.

Der mögliche toxische Effekt von Statinen auf die Muskulatur ist seit langem bekannt. Während es in einigen Fällen lediglich zu einem asymptomatischem Anstieg der CK im Serum kommt, können Statine bei anderen Patienten zu einer Rhabdomyolyse führen. Im Jahr 2007 wurde jedoch erstmals eine Gruppe von Patienten beschrieben, bei denen es auch nach Absetzen der Statine zu einer progrediente Muskelschwäche kam [20]. Es wird angenommen, dass Statine, durch die Enzymhemmung im Muskel neben direkt myotoxischen Effekten auch autoimmunologische Prozesse einschließlich der Bildung von Auto-Antikörpern triggern können. Unterstützt wird diese Hypothese durch die Identifikation spezifischer Antikörper gegen die 3-hydroxy-3-methylglutaryl-Coenzym A Reduktase (antiHMGCoAR) [8]. Diese Auto-Antikörper lassen sich bei bis zu $60 \%$ der NM-Patienten im Serum nachweisen. Bei etwa zwei Drittel der Patienten mit nachweisbaren HMGCoAR-Antikörpern besteht eine Assoziation mit der vorherigen Einnahme von Statinen.

Eine Vielzahl weiterer NM-Patienten zeigt Antikörper gegen das single recognition particle (SRP), welche mit einer schlechteren Prognose assoziiert sind [21].

\section{Myositis bei Overlap-Syndrom („Overlap Myositis“)}

Bei einer Vielzahl von Patienten mit Myositis lassen sich extramuskuläre Symptome und Organbeteiligungen nachweisen, die auf das Vorliegen einer systemischen Kollagenose hindeuten. Hierzu zählen bspw. Raynaud-Symptomatik, Sicca-Syndrom, Arthritis, Hautveränderungen und Serositis. Andererseits können auch Patienten, die primär an einer Kollagenose leiden, im Krankheitsverlauf über muskuläre Symptome klagen.

Die Charakterisierung von Overlap-Syndromen hat sich auch durch die Identifikation von „Myositis-assoziierten“ Auto-Antikörpern verändert. Die häufigste Form einer Myositis bei Overlap-Syndrom („Overlap Myositis“) geht mit einer systemischen Sklerose einher zusammen mit histologischen Muskelveränderungen, die einer PM oder DM gleichkommen [22]. Bei diesen Patienten lassen sich häufig Anti-PmScl und Anti-Ku Antikörper nachweisen.

Für das Overlap-Syndrom, welches mit einem Sjögren-Syndrom assoziiert ist, sind oft Anti-SSA/Ro und Anti-SSB/La Antikörper nachweisbar. Aufgrund der Häufigkeit eines Overlap-Syndroms sollte eine Myositis idealerweise immer in enger Zusammenarbeit von Neurologen, Rheumatologen und Dermatologen behandelt werden.

\section{Einschlusskörpermyositis}

Die IBM nimmt unter den inflammatorischen Myopathien eine Sonderstellung ein, da sich neben entzündlichen Veränderungen, auch degenerative Veränderungen finden lassen. Gleichzeitig ist sie die häufigste entzündliche Myopathie bei Patienten über 45 Jahre [23].
Die Prävalenz wird zwischen 4,5 und 9,5/1 Million Einwohnern geschätzt und steigt mit zunehmendem Alter weiter an. Im Gegensatz zur DM, PM und NM findet sich bei der IBM eine Bevorzugung des männlichen Geschlechts, welches 3-mal häufiger von der Erkrankung betroffen ist als das weibliche. Zudem unterscheidet sich die IBM von den anderen inflammatorischen Myopathien hinsichtlich des klinischen Bildes: Die Muskelschwäche und -atrophien entwickeln sich deutlich langsamer als bei den anderen Myositiden und erstrecken sich über einen Zeitraum von Monaten bis Jahren und zeigen typischerweise ein asymmetrisches Verteilungsmuster. Häufig betroffene Muskelgruppen sind die Fingerflexoren, Kniestrecker und Fußheber mit ausgeprägter Atrophie der Unterarm- und Quadricepsmuskulatur. Darüber hinaus berichten bis zu 60 \% der Patienten über eine Schluckstörung im Zuge der Beteiligung der Pharynxmuskulatur [24]. Insbesondere vor dem Hintergrund möglicher, schwerwiegender Komplikationen, wie Bolusaspiration und Aspirationspneumonie, sollte der Untersucher ein besonderes Augenmerk auf das Vorliegen einer Schluckstörung richten und eine zielgerichtete Anamnese führen. Die Erkrankung verläuft über Jahre langsam progredient und führt im Mittel nach 10 Jahren zur Rollstuhlpflichtigkeit der Patienten. Laborchemisch finden sich nur leicht- bis mittelgradig erhöhte CK-Werte im Serum der Patienten.

Typische Charakteristika der IBM finden sich in der Muskelbiopsie. Neben dem Nachweis endomysialer inflammatorischer Infiltrate aus CD8 + T-Zellen und Makrophagen sowie einer vermehrten MHC-I Expression, lassen sich auch degenerative Mechanismen erkennen. So finden sich in der Muskelbiopsie von IBM-Patienten auch congophile Amyloid-Ablagerungen, Einschlusskörper und Vakuolen. Die genaue Ätiologie und das Zusammenspiel von Inflammation und Degeneration sind weiterhin unklar. Postuliert wird derzeit, dass eine entzündungsmediierte Hochregulation von Amyloid-Vorläuferprotein (APP) und $\beta$-Amyloid zu vermehrter Ablagerung, damit verbundenem oxidativen Zellstress und Autophagie und somit schlussendlich zum Zelltod führen [25-27].

Die Diagnose der IBM wird anhand kürzlich überarbeiteter klinisch-pathologischer Kriterien gestellt, die im 188. ENMC-Workshops von der ENMC IBM Working Group formuliert wurden. Dabei wird unterschieden zwischen einer klinisch-pathologisch definierten, klinisch definierten und wahrscheinlichen IBM ( $\triangleright$ Tab. 3) (Daten aus [28]). Gegenüber früheren Diagnosekriterien findet nun auch der klinische Phänotyp eine größere Beachtung, wodurch häufig eine frühere Diagnose möglich wird.

Neben dem Phänotyp und den Besonderheiten der Muskelbiopsie, unterscheidet sich die IBM von den anderen inflammatorischen Myopathien insbesondere auch hinsichtlich des fehlenden Ansprechens auf eine immunsuppressive Therapie. Die Therapieoptionen der IBM werden daher gesondert besprochen.

\section{Therapie}

Aufgrund der Seltenheit von Myositiden und des damit verbundenen Mangels an hochwertigen klinisch-kontrollierten Studien, basieren die Therapieempfehlungen von entzündlichen Myopathien überwiegend auf Expertenempfehlungen und kleineren Studien.

- Abb. 1 gibt eine Übersicht über den gängigen Therapiealgorithmus von PM, DM und NM. Die Therapie der Myositis bei Over- 
- Tab. 3 „ENMC IBM Research Diagnostic Criteria 2011“ - Daten aus [28].

\begin{tabular}{|c|c|c|c|}
\hline & Klassifikation & & \\
\hline & Klinisch-pathologisch definierte IBM & Klinisch definierte IBM & Wahrscheinliche IBM \\
\hline $\begin{array}{l}\text { Klinische Befunde } \\
\text { und Laborparameter }\end{array}$ & $\begin{array}{l}\text { - Dauer }>12 \text { Monate } \\
\text { - Alter bei Erkrankung > } 45 \text { Jahre } \\
\text { - Kniestreckerschwäche } \geq \text { Schulterab- } \\
\text { duktionsschwäche und/oder } \\
\text { Fingerflexorschwäche > Schulterab- } \\
\text { duktionsschwäche } \\
\text { - } \text { sCK nicht höher als } 15 x \text { der Norm }\end{array}$ & $\begin{array}{l}\text { - Dauer }>12 \text { Monate } \\
\text { - } \text { Alter bei Erkrankung > } 45 \text { Jahre } \\
\text { - } \text { Kniestreckerschwäche } \geq \text { Schulterab- } \\
\text { duktionsschwäche und Fingerflexor- } \\
\text { schwäche > Schulterabduktions- } \\
\text { schwäche } \\
\text { - } \text { sCK nicht höher als } 15 x \text { der Norm }\end{array}$ & $\begin{array}{l}\text { - Dauer }>12 \text { Monate } \\
\text { - Alter bei Erkrankung > } 45 \text { Jahre } \\
\text { - Kniestreckerschwäche } \geq \\
\text { Schulterabduktionsschwäche oder } \\
\text { Fingerflexorschwäche > } \\
\text { Schulterabduktionsschwäche } \\
\text { - sCK nicht höher als } 15 x \text { der Norm }\end{array}$ \\
\hline $\begin{array}{l}\text { Pathologische } \\
\text { Merkmale }\end{array}$ & $\begin{array}{l}\text { Alle der folgenden: } \\
\text { - endomysiale inflammatorische } \\
\text { Infiltrate } \\
\text { - umrandete („rimmed“) Vakuolen } \\
\text { - Protein-Akkumulation * oder } \\
\text { 15-18nm Filamente }\end{array}$ & $\begin{array}{l}\text { Ein oder mehrere, aber nicht alle, der } \\
\text { folgenden: } \\
\text { - endomysiale inflammatorische } \\
\text { Infiltrate } \\
\text { - Hochregulation von MCH Klasse I } \\
\text { - umrandete („rimmed“) Vakuolen } \\
\text { - Protein-Akkumulation * oder } \\
\text { 15-18nm Filamente }\end{array}$ & $\begin{array}{l}\text { Ein oder mehrere, aber nicht alle, der } \\
\text { folgenden: } \\
\text { - endomysiale inflammatorische } \\
\text { Infiltrate } \\
\text { - Hochregulation von MHC Klasse I } \\
\text { - umrandete („rimmed“) Vakuolen } \\
\text { - Protein-Akkumulation * oder } \\
\text { 15-18nm Filamente }\end{array}$ \\
\hline
\end{tabular}

lap-Syndrom erfolgt analog zu den anderen Myositisformen, jedoch unter Berücksichtigung der Mitbehandlung einer assoziierten SLE, Sklerodermie, Arthritis usw.

\section{Therapie der PM, DM und NM}

\section{Glukokortikoide}

In der Akuttherapie der PM, DM und NM sind hochdosierte Glukokortikoide Mittel der ersten Wahl. In der Regel erfolgt zunächst die intravenöse Gabe von Methylprednisolon in einer Dosis von 500-1 000 mg täglich über einen Zeitraum von 3-5 Tagen. Anschließend wird die Therapie oral in einer Dosis von $1 \mathrm{mg} / \mathrm{kg}$ Körpergewicht bis zur klinischen Besserung fortgeführt. Eine zu frühe Reduktion der Prednisolondosis sollte vermieden werden und erst nach einem Behandlungszeitraum von mindestens 8 Wochen bzw. sicherem Ansprechen der Therapie erfolgen. Dabei hat sich bewährt, die Dosis alle 2-4 Wochen um $10 \mathrm{mg}$ bis auf eine Zieldosis von zunächst $20 \mathrm{mg}$ täglich zu reduzieren. Ab dieser Dosis sollte die Reduktion in $2,5 \mathrm{mg}$ bis $5 \mathrm{mg}$-Schritten erfolgen. Insgesamt sollte das Ausschleichen der Prednisolondosis unter engmaschiger Kontrolle des klinischen Krankheitsbildes erfolgen. In den meisten Fällen ist eine Erhaltungsdosis zwischen 5-10 mg täglich notwendig. Als Alternative zur täglichen Prednisolon-Einnahme kommt eine alternierende Gabe alle 2 Tage in Betracht. Bei längerer Therapiedauer sollte begleitend eine Medikation zur Osteoporoseprophylaxe und Magenschutz erfolgen.

Da viele Patienten nach initial gutem Ansprechen auf die Prednisolon-Therapie eine erneute Verschlechterung zeigen und in Anbetracht des Nebenwirkungsprofils einer Langzeit-Kortikoidmedikation, wird häufig die Verabreichung eines zusätzlichen Immun- suppressivums notwendig. In der Langzeit-Therapie sollte die Prednisolondosis nach Möglichkeit unter 7,5 mg/Tag liegen.

\section{Immunsupressiva}

Verschiedene Immunsuppressiva kommen in der Erhaltungs- und Langzeittherapie der PM, DM und NM zum Einsatz. Ziel der Behandlung ist eine andauernde Stabilisierung der Erkrankung und Rezidivprophylaxe sowie die Einsparung der Kortikoiddosis.

Azathioprin kommt in der DM, PM und NM am häufigsten parallel zu Glukokortikoiden zum Einsatz. Unter Berücksichtigung der individuellen TPMT-Aktivität und engmaschiger Kontrolle des Differenzialblutbildes und der Leberwerte, erfolgt die Eindosierung üblicherweise in einer Dosis bis $3 \mathrm{mg} / \mathrm{kg}$ Körpergewicht. Dabei orientiert sich die Zieldosis an den Lymphozytenwerten, deren Zielbereich mit 600-1000/ $\mu$ l definiert ist.

Als Alternative zum Azathioprin kommen Mofetilmycophenolat (MMF), Ciclosporin und Methotrexat (MTX) zum Einsatz. Die Zieldosis von MMF liegt in der Regel bei einer Tagesdosis von $2 \mathrm{~g}$ verteilt auf 2 Einzeldosen. Die Therapie sollte, wie Azathioprin, unter regelmäßiger Kontrolle des Blutbildes erfolgen.

MTX zeigt gegenüber Azathioprin den möglichen Vorteil eines schnelleren Wirkungseintritts und wird üblicherweise in einer Dosis von 7,5-20 mg einmal pro Woche eingesetzt. Parallel sollte eine Folsäuresubstitution am Tag nach der MTX-Gabe erfolgen. Bei myoglobinurischem Nierenversagen besteht die Gefahr einer MTX-Kumulation und Entwicklung einer MTX-Pneumonitis.

Ciclosporin erfordert in Anbetracht der dosisabhängigen Nephrotoxizität ein hohes Maß an Patienten-Compliance und wird daher eher als Reservemedikament eingesetzt. Die Dosis liegt bei 

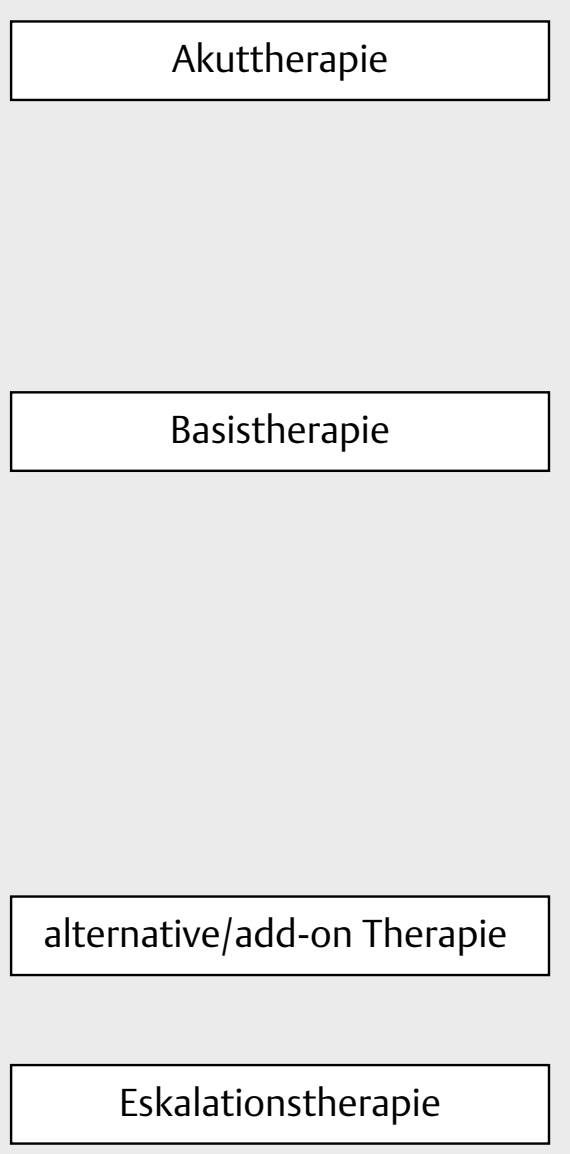

- orales Prednisolon mit $1 \mathrm{mg} / \mathrm{kg}$ Körpergewicht für mind. 8 Wochen bzw. bis zum Ansprechen

- ggf. initial intravenöse Methylprednisolon Pulstherapie mit 500-1000 mg/Tag über 3-5 Tage

- vorsichtige Dosisreduktion um $10 \mathrm{mg}$ alle 2 - 4 Wochen, ab $20 \mathrm{mg}$ Tagesdosis um 2,5 - $5 \mathrm{mg}$

- Prednisolon in Erhaltungsdosis von 5-10 mg/Tag

- Immunsuppressivum bei fehlendem Ansprechen bzw. zur Einsparung der Prednisolondosis

- Azathioprin - Dosis ca. $3 \mathrm{mg} / \mathrm{kg}$ Körpergewicht (Cave: TPMT) gemäß

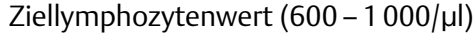

- Mofetilmycophenolat $1000 \mathrm{mg}$ bis $2000 \mathrm{mg} / \mathrm{Tag}$

- MTX - 7,5-20 mg wöchentlich (parallel: Folsäuresubstitution)

intravenöse Immunglobuline, initial $2 \mathrm{~g} / \mathrm{kg}$ Körpergewicht; danach $1 \mathrm{~g}(-2 \mathrm{~g}) / \mathrm{kg}$ Körpergewicht alle 4-6 Wochen

- Rituximab - z. B. 2 x $1000 \mathrm{mg}$ in 14-tägigem Abstand, ggf. Wiederholung nach 6-9 Monaten

- Cyclophosphamid - z.B. i. v. Pulstherapie mit $0,5-1,0 \mathrm{~g} / \mathrm{m}^{2}$ Körperoberfläche, initial 6 Zyklen alle 4 Wochen gemäß Leukozytennadir nach $10 \mathrm{~d}$

- Abb. 1 Therapiealgorithmus der PM, DM und NM.

2,5-5 mg/kg Körpergewicht und sollte unter regelmäßigen Plasmaspiegelkontrollen und Prüfung der Nierenfunktion gesteuert werden.

Bei schweren Verläufen von PM, DM und NM hat sich im klinischen Alltag Rituximab bewährt, ein monoklonaler anti-CD20 Antikörper, der zur B-Zell-Depletion führt und entsprechende Effekte in einer klinischen Studie [29] sowie diversen Fallserien geboten hat (Übersicht in [1-3]). Rituximab sollte 2-malig im Abstand von 14 Tagen in einer Dosis von 1000 mg verabreicht werden. Eine Wiederholung der Therapie findet entsprechend des klinischen Ansprechens bzw. nach 6-9 Monaten erneut statt. Bei schwerwiegender extramuskulärer Organbeteiligung bzw. auch einer vaskulitischen Genese kann Cyclophosphamid zur Eskalationstherapie verwendet werden. Cyclophosphamid kann oral verabreicht werden, wird jedoch wegen eines günstigeren Risiko-/Nutzenprofils üblicherweise als intravenöse Pulstherapie mit initial 6 Pulsen alle 4 Wochen intravenös in einer Dosis von 500-1000 mg/m² Körperoberfläche appliziert. Die Dosiseinstellung erfolgt anhand der Dokumentation des Leukozytennadirs nach 10 Tagen.
Bei allen Immunsuppressiva kann mit einem steroidsparenden Wirkungseintritt erst nach einigen Wochen bis Monaten gerechnet werden, so dass frühzeitig über einen entsprechenden Therapiebeginn nachgedacht werden sollte.

\section{Intravenöse Immunglobuline (IVIg)}

Bei fehlendem Ansprechen auf Glukokortikosteroide und Immunsuppressiva bzw. im Falle einer Unverträglichkeit stellt die intravenöse Immunglobulingabe eine wichtige Therapieoption dar [30], die auch vom gemeinsamen Bundesausschuss als verordnungsfähig befunden wurde (www.g-ba.de). Die Gabe erfolgt initial mit $2 \mathrm{~g} / \mathrm{kg}$ und danach mit $1 \mathrm{~g} / \mathrm{kg}$ (bis $2 \mathrm{~g} / \mathrm{kg}$ ) alle 4-6 Wochen, verteilt über 1-5 Tage. Entsprechend des klinischen Ansprechens, sollte dann eine sukzessive Dosisreduktion bis zur individuellen Erhaltungsdosis erfolgen. Gegebenenfalls kann im Verlauf auch eine Streckung des Therapieintervalls erwogen werden. Nach einer Stabilisierungsphase kann individuell ein Aussetzen der IVIg-Therapie diskutiert werden. 
Während die Gabe von IVIg beim Erwachsenen vor allem als als add-on Therapie bzw. Therapiealternative erfolgt, kommt diese bei der juvenilen DM häufig deutlich früher zum Einsatz. Hinsichtlich des Nebenwirkungsprofils bietet die IVIg-Therapie deutliche Vorteile gegenüber immunsuppressiven Therapien. Aus diesem Grund und bei fehlender Teratogenität finden IVIg ihren Einsatz auch bei DM-, PM- oder NM-Patientinnen mit Kinderwunsch oder bestehender Schwangerschaft.

\section{Neuere Therapiestrategien}

Der Einsatz verschiedener experimenteller Therapiealternativen wurde in einzelnen Fallberichten über therapie-refraktäre MyositisPatienten beschrieben. Zum Einsatz kamen hierbei bspw. TNF- $\alpha$ Inhibitoren, wie Etanercept und Infliximab. Für diese Substanzen wurde jedoch sogar teilweise eine Exazerbation der Erkrankung beschrieben, sodass von ihrem Einsatz zum jetzigen Zeitpunkt abgesehen werden sollte [31,32].

Weitere Therapieansätze beinhalten den Einsatz von IFN- $\alpha$ Antagonisten wie Sifalimumab, den CD52-Antikörper Alemtuzumab oder Zytokininhibitoren wie z. B. für IL-6 oder IL-1 $\beta$. Mit einigen der entsprechenden monoklonalen Antikörpern sind bereits Konzeptstudien durchgeführt worden bzw. befinden sich bereits klinische Prüfungen in Vorbereitung bzw. in Arbeit.

Bei Patienten mit interstitieller Lungenerkrankung und Myositis (Overlap Syndrom) konnte in einer retrospektiven Studie ein positiver Effekt für den Einsatz von Tacrolimus im Hinblick auf die Überlebenszeit im Vergleich zur konventionellen Therapie mit Prednisolon, intravenöser Gabe von Cyclophosphamid und/oder Ciclosporin gezeigt werden [33].

\section{Therapie der IBM}

Die Behandlung der IBM stellt nach wie vor eine große Herausforderung dar, da fast sämtliche Therapieansätze ohne Effekt bleiben. Nur in wenigen Einzelfällen wurde ein gewisser Effekt auf die Muskelkraft oder Krankheitsaktivität für immunsuppressive Therapien und IVIg beschrieben (Übersicht in [34]). Eine Hypothese für das fehlende Ansprechen auf die herkömmlichen Therapieansätze, ist das Fortbestehen der degenerativen Mechanismen bei dieser Erkrankung. Möglicherweise ist bei vielen Patienten die hierdurch bedingte Muskelschädigung zum Zeitpunkt der Erstvorstellung schon so weit fortgeschritten, dass herkömmliche immunsuppressive Therapiestrategien nicht mehr greifen können [35]. Pilot-Studien zum Einsatz von Anti-Thymozyten-Globulin (ATG) und MTX oder dem monoklonalen Antikörper Alemtuzumab konnten allenfalls eine passagere Stabilisierung nachweisen [36, 37]. Die Ergebnisse sind jedoch insbesondere für Alemtuzumab vorsichtig zu interpretieren, da das Design der Studie nicht placebo-kontrolliert war. Auch für den Einsatz von Interferon $\beta$-1a, Oxandrolon und Simvastatin konnte kein positiver Effekt dokumentiert werden [38-41].

Eine Reihe von Studien untersuchte den Einsatz von intravenösen Immunglobulinen bei IBM-Patienten. Wenngleich eine transiente Stabilisierung der Kraft beschrieben wurde, erreichten die Ergebnisse lediglich eine signifikant verbesserte Schluckfunktion [42, 43] bzw. Besserung der Alltagsfähigkeiten [44]. In einer weiteren Studie bei IBM-Patienten mit assoziierter Schluckstörung zeigte sich ebenfalls eine signifikante Besserung der Dysphagie unter IVIg-Therapie [45]. Die Datenlage zum Einsatz von IVIg in der Therapie der IBM ist somit widersprüchlich. Eine kürzlich veröffentlichte Cochrane-Analyse zur IBM fasst zusammen, dass keine eindeutigen Schlüsse aus den vorhanden Studien gezogen werden können [46]. Aufgrund der kleinen Fallzahlen und der kurzen Beobachtungszeiträume lässt sich keine sichere Aussage hinsichtlich der Wirksamkeit der untersuchten Präparate für die IBM treffen. Größere und qualitativ hochwertigere Studien mit längerem Studienzeitraum von z. B. einem Jahr sind notwendig, um therapeutische Veränderungen bei der IBM verlässlich detektieren zu können. Insbesondere auch die IVIg Studien sind mit 3 Monaten Dauer deutlich zu kurz, sodass ein negativer Studieneffekt nicht sicher abzuleiten ist.

Unter Berücksichtigung des individuellen Krankheitsverlaufs, verschiedener Fallberichte und klinischer Expertenmeinung sowie der aktuellen Leitlinien zur Therapie der Myositis (www.awmf.org: Therapieleitlinie Myositis) erscheint ein Therapieversuch mit IVIg für die Dauer von 6 Monaten gerechtfertigt. Die Therapie sollte zunächst in einer Dosis von $2 \mathrm{~g} / \mathrm{kg}$ Körpergewicht, verteilt über 2-5 Tage erfolgen. Im Anschluss sollte eine Dosisreduktion auf $1 \mathrm{~g} / \mathrm{kg}$ Körpergewicht stattfinden. Das Therapieintervall liegt analog zur DM und PM bei 6 bis längstens 8 Wochen. 6 Monate nach Therapiebeginn sollte dann eine sorgfältige Reevaluation und Beurteilung des klinischen Ansprechens erfolgen.

\section{Nicht medikamentöse Therapien}

Neben medikamentösen Therapieregimen haben auch physiotherapeutische Maßnahmen einen relevanten Stellenwert in der Behandlung inflammatorischer Myopathien.

Mehrere kleinere Untersuchungen konnten belegen, dass Myositis-Patienten von regelmäßigen physiotherapeutischen Maßnahmen und Krafttraining profitieren [47][48]. Entgegen vorheriger Annahmen konnten kein schädlicher Effekt von regelmäßigem Training auf die Muskelkraft oder Zeichen einer verstärkten Muskelschädigung nachgewiesen werden [49]. Regelmäßige Physiotherapie und rehabilitative Maßnahmen sollten daher ein fester Bestandteil der nicht medikamentösen Therapie von MyositisPatienten sein.

Im Falle einer oropharyngealen Beteiligung und damit verbundenen Schluckstörung sollten Patienten, nach einer eingehenden phoniatrischen Mitbeurteilung des Schluckaktes mittels FEES, eine engmaschige logopädische Betreuung erhalten. Insbesondere das Erlernen von Kompensationsmechanismen und eine Beratung bezüglich der Nahrungs- und Flüssigkeitskonsistenz sollten dabei im Vordergrund stehen. Oberstes Ziel ist hierbei die Verhinderung von Komplikationen wie einer Aspiration und der oft damit verbundenen Pneumonie. Da es bei einer IBM zu einer funktionellen Enge im Bereich des oberen Ösophagussphinkters (M. cricopharyngeus) kommt [50], können lokale Therapien wie mit Botulinumtoxin oder sogar eine irreversible Myotomie erwogen werden (Übersicht in $[2,51])$. Die Indikation und Durchführung solcher individuellen Heilversuche sollte bevorzugt an erfahrenen Neuromuskulären Zentren erfolgen. 


\section{Zusammenfassung}

Die inflammatorischen Myopathien sind eine Gruppe heterogener, erworbener Muskelerkrankungen. Man unterscheidet zwischen der Polymyositis, Dermatomyositis, nekrotisierender Myopathie, Einschlusskörpermyositis und Myositis bei Overlap-Syndrom. Für die klinisch-pathologische Differenzierung der unterschiedlichen Subtypen sind eine genaue klinische Untersuchung, die Bestimmung von „Myositis spezifischen/-assoziierten“ Auto-Antikörpern sowie die Durchführung einer Muskelbiopsie unerlässlich. Insbesondere im Hinblick auf die Auswahl einer geeigneten Therapie und Einschätzung der Prognose sollte eine sorgfältige Diagnostik erfolgen. Dabei ist insbesondere auch auf eine extramuskuläre Organbeteiligung, insbesondere die interstitielle Lungenerkrankung sowie die bekannte Assoziation von inflammatorischen Myopathien mit Tumorerkrankungen zu achten. Ein zusätzliches Augenmerk sollte zudem auf dem möglichen Vorliegen einer Schluckstörung liegen, um potentiell schwere Komplikationen, wie die Aspirationspneumonie zu vermeiden.

Bei der PM, DM, NM und dem Overlap-Syndrom fokussiert sich die medikamentöse Therapie auf eine suffiziente Immunsupression. Für die IBM konnte bisher noch keine effiziente Therapie identifiziert werden; sie scheint gegenüber den herkömmlichen Therapieansätzen refraktär zu sein und ein Therapieversuch mit IVIg erscheint sinnvoll und entspricht den interdisziplinären Therapieleitlinien zur Myositis (Rheumatologie, Dermatologie und Neurologie: www.awmf.org -> Leitlinie Myositis). Für alle inflammatorischen Myopathien sollte auch die nicht-medikamentöse Therapie mit regelmäßiger Physiotherapie und einer suffizienten supportiven Therapie entsprechend berücksichtigt werden.

\section{Interessenkonflikt}

JS hat Honorare, Forschungsunterstützungen oder Reisekostenunterstützungen von Bayer, Biomarin, Biotest, CSL Behring, Grifols, Novartis, Octapharma und VitalAire erhalten.

\section{Literatur}

[1] Dalakas MC. Inflammatory muscle diseases. N Engl J Med 2015; 372 : 1734-1747

[2] Carstens P-O, Schmidt J. Diagnosis, pathogenesis and treatment of myositis: recent advances. Clin Exp Immunol 2014; 175: 349-358

[3] Breithaupt M, Schmidt J. Inflammatorische Muskelerkrankungen: Diagnostik und Therapie von Myositiden. Neurotransmitter 2014; 12: 46-56

[4] Bohan A, Peter JB. Polymyositis and dermatomyositis (first of two parts). N Engl J Med 1975; 292: 344-347

[5] Bohan A, Peter JB. Polymyositis and dermatomyositis (second of two parts). N Engl J Med 1975; 292: 403-407

[6] Targoff IN, Mamyrova G, Trieu EP et al. A novel autoantibody to a $155-k d$ protein is associated with dermatomyositis. Arthritis Rheum 2006; 54: 3682-3689

[7] Sato S, Kuwana M, Fujita T et al. Anti-CADM-140/MDA5 autoantibody titer correlates with disease activity and predicts disease outcome in patients with dermatomyositis and rapidly progressive interstitial lung disease. Mod Rheumatol 2013; 23: 496-502
[8] Mammen AL, Chung T, Christopher-Stine L et al. Autoantibodies against 3-hydroxy-3-methylglutaryl-coenzyme $A$ reductase in patients with statin-associated autoimmune myopathy. Arthritis Rheum 2011; 63: 713-721

[9] Pluk H, van Hoeve BJA, van Dooren SH] et al. Autoantibodies to cytosolic 5'-nucleotidase $1 \mathrm{~A}$ in inclusion body myositis. Ann Neurol 2013; 73 : 397-407

[10] Larman HB, Salajegheh M, Nazareno R et al. Cytosolic 5'-nucleotidase $1 \mathrm{~A}$ autoimmunity in sporadic inclusion body myositis. Ann Neurol 2013; 73: 408-418

[11] Adams EM, Chow CK, Premkumar A et al. The idiopathic inflammatory myopathies: spectrum of MR imaging findings. Radiographics 1995; 15: 563-574

[12] Tomasová Studynková J, Charvát F, Jarosová K et al. The role of MRI in the assessment of polymyositis and dermatomyositis. Rheumatol (Oxford) 2007; 46: 1174-1179

[13] Callen JP. Dermatomyositis. Lancet 2000; 355: 53-57

[14] Gerami P, Schope JM, McDonald L et al. A systematic review of adult-onset clinically amyopathic dermatomyositis (dermatomyositis siné myositis): a missing link within the spectrum of the idiopathic inflammatory myopathies. J Am Acad Dermatol 2006; 54: 597-613

[15] Bendewald M], Wetter DA, Li X et al. Incidence of dermatomyositis and clinically amyopathic dermatomyositis: a population-based study in Olmsted County, Minnesota. Arch Dermatol 2010; 146: 26-30

[16] Dalakas MC, Hohlfeld R. Polymyositis and dermatomyositis. Lancet 2003; 362: 971-982

[17] Greenberg SA. Type 1 interferons and myositis. Arthritis Res Ther 2010; 12 (Suppl 1): S4

[18] De Paepe B, Creus KK, De Bleecker JL. The tumor necrosis factor superfamily of cytokines in the inflammatory myopathies: potential targets for therapy. Clin Dev Immunol 2012; 2012: 369432

[19] Liang C, Needham M. Necrotizing autoimmune myopathy. Curr Opin Rheumatol 2011; 23: 612-619

[20] Needham M, Fabian V, Knezevic W et al. Progressive myopathy with up-regulation of MHC-I associated with statin therapy. Neuromuscul Disord 2007; 17: 194-200

[21] Hengstman GJD, Laak HJ, ter Vree Egberts WTM et al. Anti-signal recognition particle autoantibodies: marker of a necrotising myopathy. Ann Rheum Dis 2006; 65: 1635-1638

[22] laccarino L, Gatto M, Bettio $S$ et al. Overlap connective tissue disease syndromes. Autoimmun Rev 2013; 12: 363-373

[23] Mastaglia FL, Needham M. Inclusion body myositis: a review of clinical and genetic aspects, diagnostic criteria and therapeutic approaches. J Clin Neurosci 2015; 22: 6-13

[24] Dimachkie MM, Barohn RJ. Inclusion body myositis. Curr Neurol Neurosci Rep 2013; 13: 321

[25] Schmidt J, Barthel K, Wrede A et al. Interrelation of inflammation and APP in sIBM: IL-1 beta induces accumulation of beta-amyloid in skeletal muscle. Brain 2008; 131: 1228-1240

[26] Muth IE, Barthel K, Bähr M et al. Proinflammatory cell stress in sporadic inclusion body myositis muscle: overexpression of alphaB-crystallin is associated with amyloid precursor protein and accumulation of beta-amyloid. J Neurol Neurosurg Psychiatry 2009; 80: 1344-1349

[27] Schmidt J, Barthel K, Zschüntzsch J et al. Nitric oxide stress in sporadic inclusion body myositis muscle fibres: inhibition of inducible nitric oxide synthase prevents interleukin- $1 \beta$-induced accumulation of $\beta$-amyloid and cell death. Brain 2012; 135: 1102-1114

[28] Rose MR.ENMC IBM Working Group. 188th ENMC International Workshop: Inclusion Body Myositis, 2-4 December 2011, Naarden, The Netherlands. Neuromuscul Disord 2013; 23: 1044-1055 
[29] Oddis CV, Reed AM, Aggarwal R et al. Rituximab in the treatment of refractory adult and juvenile dermatomyositis and adult polymyositis: a randomized, placebo-phase trial. Arthritis Rheum 2013; 65: 314-324

[30] Gordon PA, Winer JB, Hoogendijk JE et al. Immunosuppressant and immunomodulatory treatment for dermatomyositis and polymyositis. Cochrane Database Syst Rev 2012; 8: CD003643

[31] Dastmalchi M, Grundtman C, Alexanderson $\mathrm{H}$ et al. A high incidence of disease flares in an open pilot study of infliximab in patients with refractory inflammatory myopathies. Ann Rheum Dis 2008; 67: 1670-1677

[32] Rouster-Stevens KA, Ferguson L, Morgan G et al. Pilot study of etanercept in patients with refractory juvenile dermatomyositis. Arthritis Care Res 2014; 66: 783-787

[33] Kurita T, Yasuda S, Oba K et al. The efficacy of tacrolimus in patients with interstitial lung diseases complicated with polymyositis or dermatomyositis. Rheumatol (Oxford) 2015; 54: 1536

[34] Breithaupt M, Schmidt J. Update on treatment of inclusion body myositis. Curr Rheumatol Rep 2013; 15: 329

[35] Dalakas MC. Immunotherapy of myositis: issues, concerns and future prospects. Nat Rev Rheumatol 2010; 6: 129-137

[36] Lindberg C, Trysberg E, Tarkowski A et al. Anti-T-lymphocyte globulin treatment in inclusion body myositis: a randomized pilot study. Neurology 2003; 61: 260-262

[37] Dalakas MC, Rakocevic G, Schmidt ] et al. Effect of Alemtuzumab (CAMPATH 1-H) in patients with inclusion-body myositis. Brain 2009; 132: $1536-1544$

[38] Muscle Study Group. Randomized pilot trial of betaINF1a (Avonex) in patients with inclusion body myositis. Neurology 2001; 57: 1566-1570

[39] Muscle Study Group. Randomized pilot trial of high-dose betalNF-1a in patients with inclusion body myositis. Neurology 2004; 63: 718-720

[40] Rutkove SB, Parker RA, Nardin RA et al. A pilot randomized trial of oxandrolone in inclusion body myositis. Neurology 2002; 58 : 1081-1087
[41] Sancricca C, Mora M, Ricci E et al. Pilot trial of simvastatin in the treatment of sporadic inclusion-body myositis. Neurol Sci 2011; 32: 841-847

[42] Dalakas MC, Sonies B, Dambrosia J et al. Treatment of inclusion-body myositis with IVIg: a double-blind, placebo-controlled study. Neurology 1997; 48: 712-716

[43] Dalakas MC, Koffman B, Fujii M et al. A controlled study of intravenous immunoglobulin combined with prednisone in the treatment of IBM. Neurology 2001; 56: 323-327

[44] Walter MC, Lochmüller $\mathrm{H}$, Toepfer $\mathrm{M}$ et al. High-dose immunoglobulin therapy in sporadic inclusion body myositis: a double-blind, placebocontrolled study. J Neurol 2000; 247: 22-28

[45] Cherin P, Pelletier S, Teixeira A et al. Intravenous immunoglobulin for dysphagia of inclusion body myositis. Neurology 2002; 58: 326

[46] Rose MR, Jones $\mathrm{K}$, Leong $\mathrm{K}$ et al. Treatment for inclusion body myositis. Cochrane Database Syst Rev 2015; 6: CD001555

[47] Alemo Munters L, Dastmalchi M, Katz A et al. Improved exercise performance and increased aerobic capacity after endurance training of patients with stable polymyositis and dermatomyositis. Arthritis Res Ther 2013; 15: R83

[48] Alexanderson H, Munters LA, Dastmalchi M et al. Resistive home exercise in patients with recent-onset polymyositis and dermatomyositis - a randomized controlled single-blinded study with a 2-year followup. J Rheumatol 2014; 41: 1124-1132

[49] Voet NBM, van der Kooi EL, Riphagen II et al. Strength training and aerobic exercise training for muscle disease. Cochrane Database Syst Rev 2013; 7: CD003907

[50] Cox FM, Verschuuren J], Verbist BM et al. Detecting dysphagia in inclusion body myositis. J Neurol 2009; 256: 2009-2013

[51] Breithaupt M, Schmidt J. Treatment strategies for inclusion body myositis. Expert Opin Orphan Drugs 2014; 2: 1255-1265 